Ana Lucia de Moura Pontes ${ }^{1}$

Sergio Rego ${ }^{2}$

Luiza Garnelo ${ }^{3}$

\title{
O modelo de atenção diferenciada nos Distritos Sanitários Especiais Indígenas: reflexões a partir do Alto Rio Negro/AM, Brasil
}

\author{
The differentiated care model in the Special Indigenous Health \\ Districts: reflections based on Alto Rio Negro \\ in Amazonia State, Brazil
}

Escola Politécnica de Saúde Joaquim Venâncio, Fiocruz. Av. Brasil 4365, Manguinhos. 21045-900 Rio de Janeiro RJ Brasil. analupontes@fiocruz.br ${ }^{2}$ Escola Nacional de Saúde Pública Sergio Arouca, Fiocruz.

${ }^{3}$ Instituto de Pesquisa Leônidas e Maria Deane, Fiocruz.
Abstract The implementation of the National Policy for Healthcare of Indigenous Peoples (Pnaspi) in the Alto Rio Negro Amazon region was analyzed based on the principles of the differentiated care model. This theme was investigated from three perspectives, namely the formulation of the guidelines, the therapeutic itineraries in indigenous villages, and the work routines of the Indigenous Community Health Agents (AIS). It involved qualitative research based on the anthropological perspective of Eduardo Menéndez. The techniques used were participant observation, interviews, and, document analysis. The formulation of the guidelines of differentiated care emphasizes the adaptation of technologies and professionals, minimizing the coexistence and disputes among healthcare models. Menéndez's perspective focused on the viewpoint of the subjects was crucial for identifying the coexistence and articulation among different forms of care in the villages through the healthcare itineraries. Nevertheless, it was not possible to identify the inclusion of indigenous health practices and representations through the work routines of the Indigenous Community Health Agents (AIS). The focus was on self-care developed and practiced by the subjects and their families.

Key words Health of indigenous people, Indigenous health services, Cultural competency, Community health workers
Resumo Analisou-se a implementação do modelo de atenção da Política Nacional de Saúde Indígena (Pnaspi) no Distrito Sanitário Especial Indígena do Alto Rio Negro, à luz da diretriz da atenção diferenciada. A temática foi investigada sob três perspectivas: formulação da diretriz; itinerários terapêuticos em aldeias indígenas; atuação dos Agentes Indígenas de Saúde (AIS). Pesquisa qualitativa, apoiada na perspectiva antropológica de Menéndez sobre modelos de atenção. A coleta de dados compreendeu observação participante, entrevistas e análise documental. A formulação da diretriz da atenção diferenciada enfatiza a adequação de tecnologias e profissionais, minimizando a dimensão da coexistência e disputas entre formas de atenção. A perspectiva de análise dos modelos de atenção desde os sujeitos, proposta por Menéndez, mostrou-se fundamental para demonstrar que a partir dos itinerários terapêuticos épossível verificar a coexistência e a articulação de diversas formas de atenção. A começar da análise das rotinas dos AIS não foi possível encontrar a inclusão ou o reconhecimento das representações e práticas indígenas de saúde. Destaca-se a autoatenção, protagonizada por sujeitos e famílias.

Palavras-chave Saúde de populações indígenas, Serviços de saúde do indígena, Noroeste amazônico, Agentes comunitários de saúde 


\section{Introdução}

A Política Nacional de Atenção à Saúde dos Povos (Pnaspi), aprovada em 2002, preconiza a implantação de um "modelo complementar e diferenciado de organização dos serviços voltados para a proteção, promoção e recuperação da saúde"1. A operacionalização da Pnaspi se dá através de 34 sistemas locais de saúde denominados Distritos Sanitários Especiais Indígenas (DSEI).

Nos DSEI, o modelo de atenção deve ser operacionalizado, em um primeiro nível, pela atuação da equipe multidisciplinar de saúde indígena (EMSI), tendo como base o trabalho dos Agentes Indígenas de Saúde (AIS) nas aldeias. Os polos -base - unidades de saúde de primeira referência para os AIS - devem contar com a presença de enfermeiros, técnicos de enfermagem, odontólogos e médicos. Fora das terras indígenas, delega-se para a Casa do Índio (Casai) a articulação com os demais serviços do Sistema Único de Saúde (SUS).

No Brasil, a concepção de Paim² sobre modelos de atenção é predominante, sendo entendida como: "formas de organização das relações entre sujeitos (profissionais de saúde e usuários) mediadas por tecnologias utilizadas no processo de trabalho em saúde"2.

Esse autor não nega, mas não reafirma a pluralidade dos contextos e sua influência nas formas de interação que possam ocorrer entre profissionais de saúde e usuários e no uso de tecnologias em saúde. Considera-se que essa conceitualização apresenta limites para discutir a operacionalização de uma diretriz fundamental da Pnaspi: a da atenção diferenciada. Tal diretriz visa adequar a prestação de serviços à variabilidade das culturas indígenas, prevendo a inclusão de racionalidades médicas diversificadas.

A perspectiva antropológica de Menéndez ${ }^{3-5}$ permite ampliar essa análise ao considerar todas as práticas utilizadas pelos grupos sociais para prevenir, diagnosticar, tratar ou aliviar um problema de saúde. Ao analisar as interações e usos das diferentes formas de atenção, esse autor permite discutir os processos de hegemonia da biomedicina, ao mesmo tempo que visibiliza a autonomia dos usuários por meio das práticas de autoatenção. Para discutir as relações de poder entre as diversas formas de atenção, será evocada a noção de poder simbólico de Bourdieu ${ }^{6,7}$. A introdução das contribuições desses dois autores pode potencializar a análise crítica da implementação da diretriz de atenção diferenciada do subsistema e trazer novas perspectivas para o debate sobre modelos de atenção no Brasil.
Neste artigo será analisada a formulação do modelo de atenção diferenciada da Pnaspi e sua operacionalização no DSEI Alto Rio Negro (DSEIRN), no Amazonas. Essa temática será investigada sob três perspectivas: a) da formulação dessa diretriz na Pnaspi; b) da organização da atenção oferecida pelo DSEIRN; c) do uso, feito pelos usuários indígenas, das formas de atenção disponíveis nas aldeias, a partir de itinerários terapêuticos ${ }^{8,9}$ seguidos pelos indígenas na busca de atendimento.

\section{Metodologia}

Menénde $z^{3-5}$ identifica os itinerários terapêuticos - aqui entendidos como os percursos percorridos pelas pessoas em busca por cuidados de saúde ${ }^{8,9}$ como os espaços em que se dá a confluência entre as práticas sanitárias autônomas da população e as dos serviços oficiais na busca de resolução dos problemas de saúde.

$\mathrm{O}$ autor afirma que os sujeitos e/ou grupos sociais utilizam potencialmente as seguintes modalidades de atenção, não excludentes ou incompatíveis entre si: Biomedicina ou medicina científica; Popular e tradicional, envolvendo curandeiros e especialistas de ervas, xamãs, benzedeiros e grupos religiosos; Alternativas , paralelas ou new age: novas religiões curativas do tipo comunitárias; Outras tradições médicas acadêmicas; Modalidades centradas na autoajuda, como as associações e grupos de portadores de patologias. Este conjunto de alternativas conforma o que o autor denomina autoatenção, entendida por ele como:

as representações e práticas que a população utiliza em nível de sujeito e grupo social para diagnosticar, explicar, atender, controlar, aliviar, aguentar, curar, solucionar ou prevenir os processos que afetam sua saúde em termos reais ou imaginários, sem a intervenção central, direta e intencional de curadores profissionais ${ }^{4}$.

É por meio da autoatenção que os sujeitos e os grupos geram articulações entre as diversas modalidades de cuidados disponíveis em seu meio social e efetuam o processo de apropriação das técnicas dos outros sistemas médicos. Os curadores profissionais também realizam apropriações das demais formas de atenção. Desse modo, a biomedicina busca a sua manutenção como forma hegemônica e mantém a subalternidade das demais formas de atenção.

A abordagem de Menéndez traz algumas implicações para a análise dos modelos de atenção 
consideradas importantes para a diretriz da atenção diferenciada no subsistema de saúde indígena. O autor enfatiza o reconhecimento da existência de diferentes formas de atenção na nossa sociedade, sendo que, nas análises das políticas de saúde no Brasil, esse pluralismo médico tem sido ignorado ou negado. Menéndez destaca:

si el sector salud quiere conocer y/o implementar el sistema de atención real que utilizan los sujetos y conjuntos sociales, debería identificar, describir y analizar las diferentes formas de atención que los conjuntos sociales manejan respecto de la variedad de padeceres reales e imaginarios que reconocen como afectando su salud ${ }^{3}$.

Ao investigar a racionalidade das ações dos sujeitos e grupos sociais na busca por cuidados, esta abordagem possibilita identificar a coexistência das formas de atenção, pressuposto da diretriz de atenção diferenciada. O autor também destaca que os processos sociais, econômicos e culturais influenciam o desenvolvimento, mudanças e relações de hegemonia/subalteridade das diferentes formas de atenção (tradicionais, biomédicas, new age), demandando uma análise específica e contextualizada dos modelos de atenção. Neste estudo serão discutidas essas implicações na análise da atenção diferenciada no subsistema de saúde indígena a partir do contexto de duas comunidades indígenas .

Para fins deste artigo, entende-se que as disputas simbólicas estabelecidas entre os diversos modos de intervenção no processo saúde-doença que subjazem ao conceito de modelo de atenção podem também ser interpretadas como manifestações do poder simbólico, tal como concebido por Bourdieu ${ }^{6,7}$. Para Bourdieu ${ }^{10}$ este tipo de poder implica o controle dos bens simbólicos e das lutas estabelecidas em torno do direito de nomear, classificar e interpretar a realidade. Ao aplicar a noção de poder simbólico às modalidades de atenção, pode-se vê-las como disputas que visam legitimar formas específicas de ver e intervir no mundo, criando ou modificando categorias de percepção que os grupos de poder tentam tornar hegemônicas e generalizáveis ao corpo social.

\section{Contexto Local do Estudo}

O trabalho de campo foi realizado em duas aldeias indígenas (Açaí e Buriti) do povo Baniwa da região do Baixo Rio Içana, afluente do Rio Negro, adscritas ao DSEIRN. Este DSEI situa-se no noroeste do estado do Amazonas, com atuação nos municípios de São Gabriel da Cachoeira, Santa Isabel do Rio Negro e Barcellos, sendo responsável pela atenção à saúde de cerca de 25 mil indígenas distribuídos em cerca 700 povoações ${ }^{11}$. Essa população se diversifica em 17 povos falantes de idiomas de três troncos linguísticos - Arawak, Tukano e Maku - além da língua geral (Nheengatu).

A aldeia Açaí, católica, tem 245 habitantes, e a aldeia Buriti, evangélica, tem 169 habitantes. Ambas têm acesso exclusivamente fluvial e são formadas basicamente por grupos de parentes que seguem o padrão tradicional de moradia da região. Na área do Baixo Içana predominam os assentamentos da etnia Baniwa, falantes da língua Nheengatu. A maior proximidade com a cidade de São Gabriel da Cachoeira (SGC) favoreceu um processo de colonização bastante contundente, com intensa atuação de missões evangélicas e católicas e outras agências da sociedade nacional, que acentuaram as transformações da sociedade tradicional Baniwa ${ }^{12}$.

Os principais informantes e tradutores foram AIS que atuam nessas aldeias. Todos os nomes utilizados são fictícios.

\section{Técnicas de pesquisa}

Esta pesquisa qualitativa é resultado de tese de doutorado, interessada no "ponto de vista do ator" como produtor de sentidos ${ }^{13-15}$. As principais técnicas utilizadas para coleta de dados foram: a) observação participante, na modalidade participante-como-observador ${ }^{16,17}$; b) entrevistas gravadas com roteiro semiestruturado ${ }^{18-20} \mathrm{com}$ agentes indígenas de saúde; c) registro sistemático em caderno de campo; d) análise documen$\mathrm{tal}^{21-23}$. A pesquisa foi aprovada na Comissão $\mathrm{Na}$ cional de Ética em Pesquisa.

A observação participante compreendeu o acompanhamento, durante 40 dias consecutivos, das atividades coletivas das aldeias e das desenvolvidas pelos AIS no seu trabalho cotidiano. A partir dessas informações, procurou-se reconstituir alguns itinerários terapêuticos dos usuários indígenas. Também foram incluídas como dado de observação participante experiências acumuladas ao longo de vários anos de vivência profissional de dois dos autores, no desenvolvimento de projeto de formação técnica dos AIS do DSEIRN, no desenvolvimento de pesquisas e outras atividades partilhadas com gestores, comunitários, profissionais e AIS.

As entrevistas realizadas com os AIS das duas aldeias priorizaram temas ligados às trajetórias de formação e ao cotidiano do trabalho. A análise documental englobou documentos oficiais da política nacional da saúde indígena. 


\section{Resultados e Discussão}

A partir da análise da Pnaspi ${ }^{1}$, do diálogo com a literatura e dos dados coletados na pesquisa, foi elaborada uma síntese, representada na Figura 1, dos seguintes elementos: princípios atribuídos à atenção diferenciada ( $1^{\mathrm{a}}$ linha); estratégias operacionais sugeridas pela Pnaspi (2 $2^{\underline{a}}$ linha); de como tem ocorrido a ação no plano local, com ênfase na atuação do AIS (3ª linha).

Segundo a Pnaspi há três princípios organizativos do modelo de atenção que se relacionam com a atenção diferenciada. O primeiro deles se refere a levar "em consideração as especificidades culturais, epidemiológicas e operacionais desses povos $^{1 "}$, o que pode ser operacionalizado mediante o desenvolvimento e uso de "tecnologias apropriadas por meio da adequação das formas ocidentais convencionais de organização de serviços ${ }^{1 "}$.

O segundo princípio sustenta a proposição de que, para a organização do modelo, deve-se levar em consideração os "sistemas de representações, valores e práticas relativas ao adoecer e buscar tratamento dos povos indígenas, bem como seus próprios especialistas ${ }^{1 "}$. Assim, indica, como estratégias operacionais, a preparação dos profissionais de saúde para a atuação em contex- to intercultural e a articulação com os sistemas tradicionais de saúde.

Considera-se como terceiro princípio a participação das lideranças e organizações indígenas e indigenistas na formulação, gestão e execução do sistema de saúde indígena por meio da estruturação de conselhos e conferências. Para além da realização do controle social, essas estruturas pretenderiam garantir a especificidade e adequação cultural das políticas e ações executadas nos Distritos.

Dados os termos da Pnaspi, observa-se que ela expressa a intenção de garantir a diferenciação da atenção da saúde indígena mediante a "adequação" de tecnologias e da atuação profissional - entendidos como partes constitutivas do modelo de atenção ${ }^{2}$-, as singularidades culturais da população atendida e a inclusão de indígenas no sistema. Nessa perspectiva, a atenção diferenciada não é entendida como elemento estruturante dos DSEI.

Retomando-se as premissas de Bourdieu, é possível afirmar que, para além de dimensões estratégicas e operacionais, ofertar atenção diferenciada significa colocar em interação diferentes visões de mundo em disputa ${ }^{6-7}$. Conforme propõe a perspectiva analítica de $\mathrm{Menendez}^{3}$, isso significa reconhecer o pluralismo médico exis-

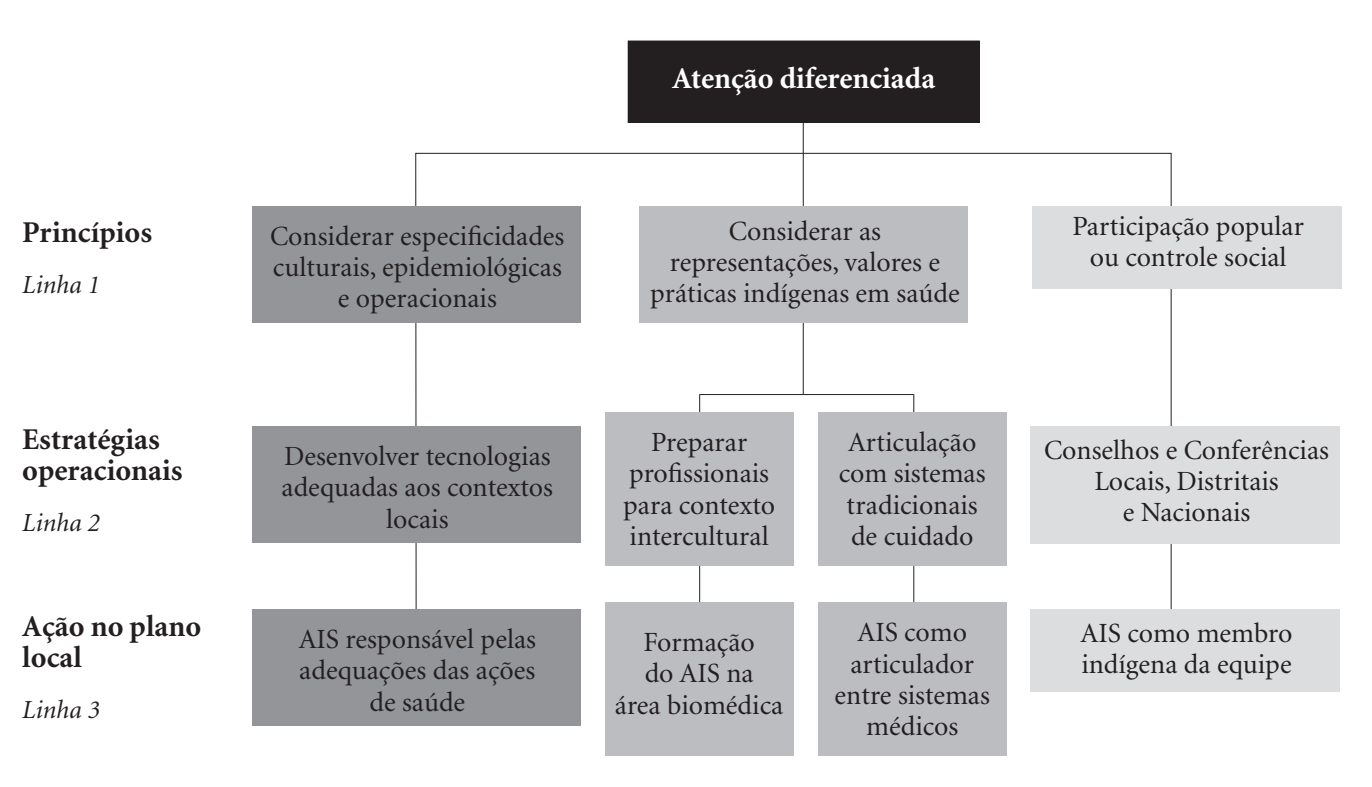

Figura 1. Síntese dos princípios, das estratégias operacionais e das ações no plano local atribuídos ao modelo de atenção diferenciada identificados na PNASPI (2002) e na literatura. 
tente nos territórios indígenas, verificar como se dá a relação entre as diferentes formas de atenção em cada contexto e identificar as racionalidades das ações dos usuários e curadores indígenas em cada Distrito.

Além da falta de uma definição sobre a atenção diferenciada, a Pnaspi sobrevaloriza a responsabilidade dos AIS para a garantia da implementação desse modelo. Caberia a esses profissionais garantir a execução das ações das equipes nas aldeias; a formação intercultural está focada na sua qualificação na área biomédica; e sua identidade indígena é usada como garantia da participação indígena no sistema. Em termos de atribuições, os documentos oficiais ${ }^{24}$ não expressam qualquer especificidade de seu trabalho por ocorrer em área indígena. Parece supor-se que, por serem indígenas, os AIS naturalmente "adequariam" tais práticas ao contexto cultural. Também ressaltamos a necessidade de problematizar se o objetivo da atenção seria somente de "adequação cultural" a um modelo já existente.

\section{A organização da atenção em duas aldeias no DSEIRN}

O modelo de atenção dos DSEI é representado em documentos oficiais, desde sua criação, pelo diagrama da Figura 2.

Essa representação visual apresenta fluxos e recursos de saúde que buscam demonstrar o funcionamento dos DSEI segundo a Pnaspi. Nela são identificados os seguintes recursos: os postos de saúde nas comunidades indígenas onde atuam os AIS; o polo-base, local de atendimento da equi-

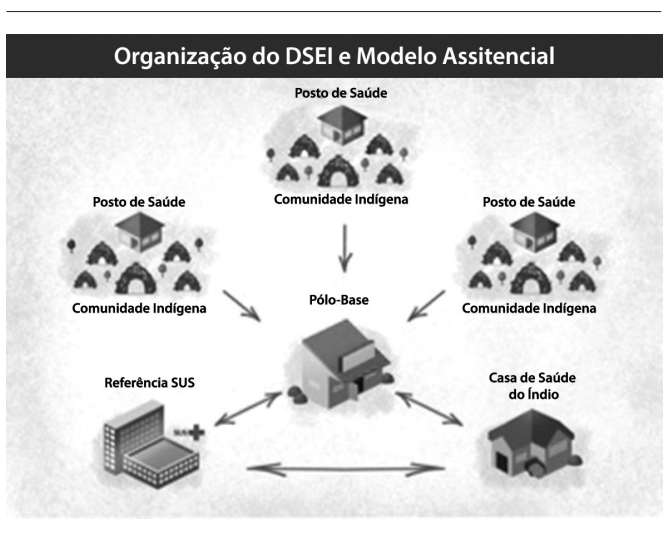

Figura 2. A Organização do modelo de atenção nos DSEI segundo PNASPI, 2012.

Fonte: SESAI/MS, 2012. pe multidisciplinar (médico, enfermeiro, técnico de enfermagem e odontólogo); e, em seguida a Rede SUS e a Casai. Também são explicitados certos fluxos que unem as aldeias indígenas aos polos-base, e entre polos-base, Casai e Rede SUS. Embora no modelo oficial tais fluxos não estejam caracterizados, é possível deduzir que expressam a circulação dos usuários indígenas nos diversos níveis do sistema de saúde. Apesar de a diretriz da atenção diferenciada referir a articulação com os sistemas tradicionais de cuidado, estes não aparecem no diagrama.

No trabalho de campo, procurou-se analisar a operacionalização desse modelo nas duas aldeias estudadas, levando em consideração todas as formas de atenção utilizadas pelos indígenas para enfrentar seus problemas de saúde ${ }^{4,5}$. Os recursos de saúde encontrados nas duas aldeias abrangiam os cuidados domésticos/familiares, relativos ao uso de plantas medicinais e/ou dietas e automedicação com remédios industrializados e outros recursos existentes nas próprias aldeias, como os terapeutas tradicionais indígenas e/ou os AIS. O corpo de agentes de saúde era formado, nessa região, por três AIS (um voluntário e dois contratados) na aldeia Açaí e um AIS contratado na aldeia Buriti - todos atendiam nos domicílios, pois não existiam postos de saúde. Com relação aos terapeutas indígenas, na aldeia de Açaí, foram referidos vários, e em Buriti somente um.

Excepcionalmente, nessa região, existia uma unidade de saúde na aldeia Açaí: um ambulatório da missão católica, que funcionava em paralelismo com DSEIRN, contando com uma médicamissionária, dois técnicos de enfermagem e uma microscopista.

Quando as medidas locais não se mostravam eficientes para a resolução dos problemas, as famílias autonomamente podiam recorrer a terapeutas tradicionais indígenas de outras aldeias ou pedir aos AIS que solicitassem à sede do DSEIRN, por radiofonia, orientação sobre procedimentos ou remoção do doente para a cidade. Por vezes, AIS de outras aldeias ou ex-agentes também eram procurados pelas famílias.

Seria ideal que o primeiro apoio aos AIS fosse o atendimento feito pelas equipes multidisciplinares de saúde do polo-base mais próximo; entretanto, a permanência de médicos e enfermeiros em área indígena carecia de continuidade. Como consequência, era frequente a remoção de enfermos para os serviços sediados na cidade. Observou-se que o grau de resolutividade intradistrital disponibilizado pelo DSEIRN era muito baixo e dependente, principalmente, da ação dos AIS. 
Na sede municipal de São Gabriel da Cachoeira (SGC), localizava-se a rede de referência (hospital e ambulatório gerenciados pelas secretarias estadual e municipal de saúde, respectivamente), para atendimento de doentes encaminhados das aldeias. Quando necessário, eles eram encaminhados à Casai, Manaus.

Esses dados subsidiaram a elaboração de uma representação visual (Figura 3), alternativa ao diagrama oficial da Sesai/MS (2012), de modo a expressar os fluxos da atenção de fato existentes nas duas aldeias estudadas. A Figura 3 sintetiza esses recursos e fluxos identificados nos itinerários terapêuticos na região e agrega a classificação das formas de atenção segundo suas características predominantes, a partir da proposta de $\mathrm{Me}$ néndez $z^{3,4}$. A direção das setas indica os movimentos dos atores sociais, enfatizando qual tendência de ação mais frequente foi observada.

Identificou-se uma diversidade de formas de atenção maior do que a apresentada na Pnaspi (Figura 2) - que no plano local incluem a bio- médica, tradicional e diversas modalidades de autoatenção -, de modo que ressalta-se o pluralismo médico, apontado por Menéndez ${ }^{3}$, no território do DSEIRN. Observou-se que o principal responsável pela busca e pelo uso das formas de atenção foram os sujeitos e suas famílias, mostrando a importância da autoatenção na organização dos cuidados. Considera-se que essa diversidade de formas de atenção e os protagonismos da autoatenção só foram possíveis de serem identificados a partir da perspectiva metodológica de Menéndez ${ }^{3}$.

Também é importante destacar os fluxos existentes no território, que podem ser entendidos em duas dimensões, assinaladas por Hannerz ${ }^{25}$ : uma espacial e outra temporal. A espacial diz respeito aos deslocamentos de objetos, pessoas e informações. Nos locais estudados, este tipo de fluxo pode ser exemplificado pela busca de um conhecedor tradicional de doenças em outra aldeia; por conversas na radiofonia entre AIS ou com a sede do DSEIRN; pelo transporte
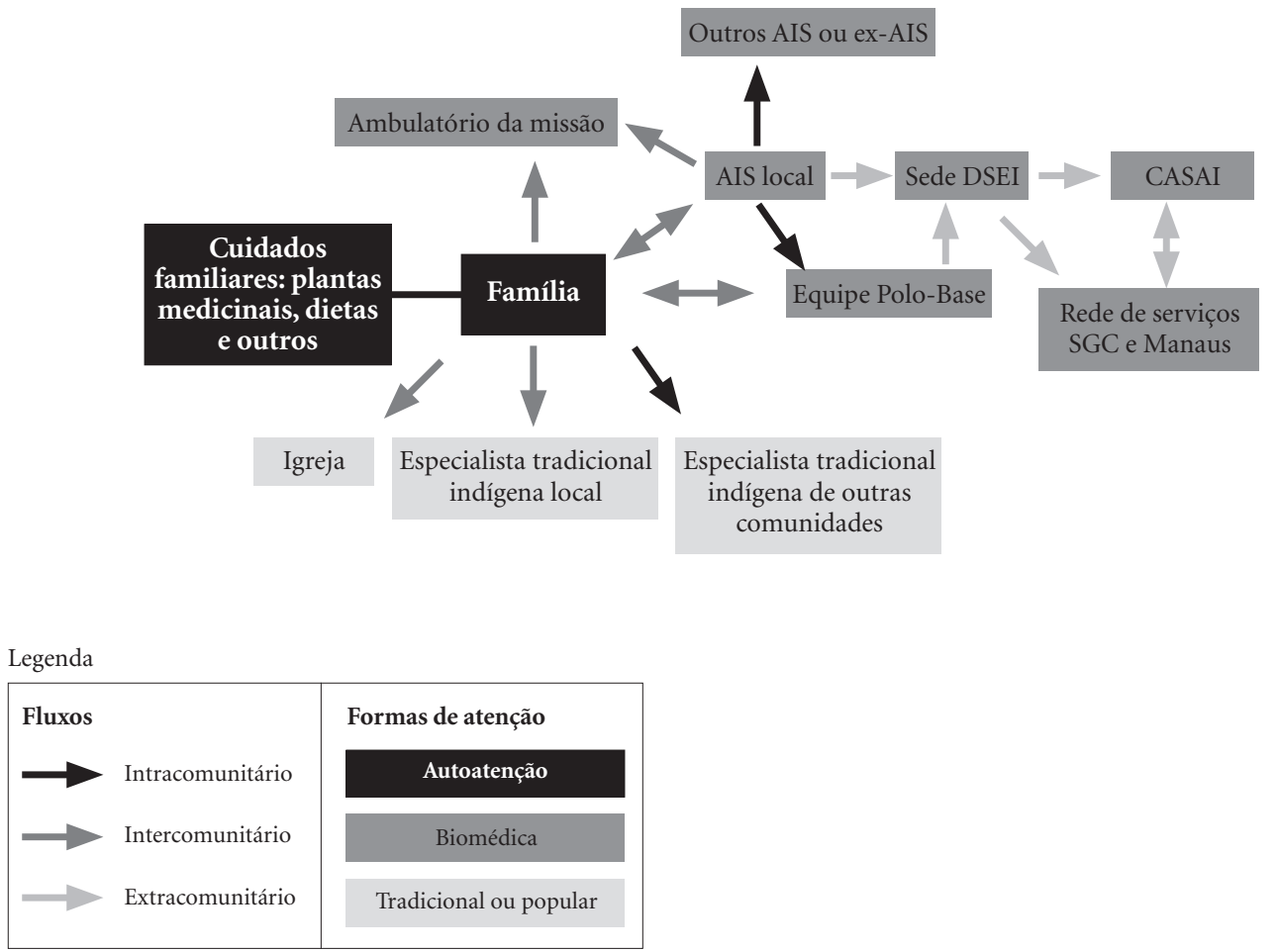

Figura 3. Organização da atenção no DSEIRN, com fluxos e recursos de saúde identificados no Baixo Içana em 2011.

Fonte: Elaboração das autoras, 2013. 
entre aldeias de medicamentos industrializados ou plantas medicinais, entre outros. Esses fluxos espaciais também são mais diversos do que os apontados na Pnaspi e possuem como principais protagonistas os sujeitos e famílias na busca por cuidado.

Segundo Hannerz ${ }^{25}$, a dimensão temporal dos fluxos se refere ao caráter processual e dinâmico da cultura, expresso, no caso, pelo movimento constante de construção sociocultural da experiência de adoecimento. Tal dinâmica permite a coexistência de diferentes sistemas terapêuticos e interpretações da doença ${ }^{26}$, tanto ocorridas no mundo atual quanto no tempo mitológico. Na realidade estudada, os sujeitos e famílias mesclam, articulam e transformam as formas de atenção disponíveis no território, conforme Menéndez ${ }^{4,5}$ indica ser feito por meio da autoatenção.

A multiplicidade de agentes terapêuticos em complexa interação na vida cotidiana (Figura 3) acena para a diversidade e a dinamicidade de conceitos e práticas que estão em diálogo no DSEIRN, em contraponto à restrita concepção do modelo oficial de assistência.

\section{A atuação dos Agentes Indígenas de Saúde (AIS)}

A observação das ações de saúde desenvolvidas no DSEIRN nas aldeias priorizou a rotina de trabalho dos AIS, pois estes permanentemente atendem a população, ao passo que os outros membros das equipes multidisciplinares realizam visitas esporádicas e irregulares. A rotina encontrada foi diferente entre as aldeias, pois em Açaí existia o ambulatório da missão católica, com presença cotidiana de médico e técnicos de enfermagem, ainda que sem vinculação com o DSEI.

Ao contrário do que foi relatado em outras realidades, onde se observa uma baixa frequência de visitas domiciliares ${ }^{27-30}$, na aldeia Açaí as visitas eram regularmente realizadas pelos AIS. Essas visitas tinham o objetivo restrito de detectar enfermos, que, quando identificados, eram encaminhados à médica no ambulatório. Somente quando a queixa levava à suspeita de gripe ou verminose, os AIS forneciam medicamento e registravam no formulário de "Produção do Agente". Entretanto, os agentes referiram que muitas pessoas não gostavam de ser atendidas no ambulatório. Assim, uma vez concluídas as visitas, os agentes passavam no ambulatório para discutir os casos com a médica. A partir do relato dos AIS, a médica realizava o diagnóstico e fornecia a medicação. Esta situação é similar aos achados de Kelly ${ }^{31}$, no atendimento aos Ianomâmi, e de $\mathrm{Novo}^{32}$, no Alto Xingu. Ambos assinalam a predominância de atendimento restrito à queixaconduta, e de pouco - ou nenhum - contato entre profissionais de saúde e pacientes indígenas.

Em Açaí, as pessoas, cujos problemas de saúde não eram resolvidos pela equipe do ambulatório, eram removidas para a cidade. Durante a única visita do restante da equipe multidisciplinar no período de coleta de dados em Açaí, a atuação dos AIS limitou-se a informar e mobilizar a aldeia para comparecer ao local. Tais atribuições foram equivalentes às observadas por outros autores, que mostram que o papel dos AIS na equipe multidisciplinar tem se limitado ao apoio logístico $^{30}$ e aos serviços gerais ${ }^{28,33}$.

Em Buriti, o trabalho dos AIS ocorria em função das demandas espontâneas da aldeia. Em geral, o AIS atendia na sua casa às pessoas que lhe procurassem com queixa de doença, e costumava ofertar algum medicamento do limitado estoque de que dispunha. Em seguida, registrava o ato no formulário de "Produção do Agente". Caso sua ação não se mostrasse eficaz, ou se concluísse ser uma doença grave, o AIS entrava em contato com a sede do DSEIRN pela radiofonia, e, se necessário, organizava a remoção para a cidade.

Outras atividades eventuais desenvolvidas pelos AIS de Buriti e de Açaí foram a dispensação de medicação para hipertensos, palestras, pesagem das crianças e preenchimento de fichas de notificação de nascidos vivos e de óbitos. Quando analisado o modelo de atenção a partir das rotinas dos AIS, fica clara a centralidade do atendimento curativo aos doentes, que só veem esse profissional como identificador de doentes, distribuidor de medicamentos e encaminhador de consultas. Essas características são identificadas como predominantes também em outros níveis do SUS ${ }^{34,35}$. O uso, pelos AIS, das taxonomias biomédicas foi recorrente na formulação dos diagnósticos, nos diálogos travados com os demais profissionais do DSEIRN e no uso de fichas e formulários. A necessidade de domínio de terminologias técnicas no trabalho cotidiano pode estar influenciando a demanda dos AIS por conhecimentos médicos, situação constatada em estudos de diversos autores ${ }^{28-30,33,36,37}$.

Assim, conforme Menéndez $z^{3}$ enfatiza, quando analisados os modelos de atenção a partir dos curadores ou profissionais, não é difícil constatar a hegemonia de uma única forma de atenção, nesse caso a biomédica, pois é predominante no sistema de saúde oficial do qual faz parte o AIS. 
Podemos dizer que a atenção diferenciada não faz parte do trabalho dos AIS e que suas rotinas são pouco diferentes daquelas adotadas em qualquer contexto urbano.

À luz dos referenciais teóricos adotados, reconhece-se que o campo da saúde é perpassado por diferentes visões de mundo em disputa ${ }^{6}$. No estudo dos campos simbólicos, Bourdieu ${ }^{6}$ sugere três instâncias nas quais as autoridades, ou o discurso oficial, operam na legalização do capital simbólico visando à imposição de um ponto de vista. No subsistema de saúde indígena, entende-se que a manutenção da autoridade biomédica como forma hegemônica de atenção se expressa: a) na instância do diagnóstico, ou seja, no reconhecimento das enfermidades; b) no discurso administrativo, que se concretiza por meio de ordens ou prescrições que organizam a prestação de serviços; c) a partir de relatórios oficiais (tais como o formulário de "Produção do Agente"). Nessas três instâncias observa-se que os AIS utilizavam categorias e formas de classificação do sistema oficial de saúde, não havendo a possibilidade de inclusão de outras formas de atenção nas suas rotinas de trabalho. Desde esse ponto de vista da implementação do subsistema, não se verifica a existência de diferentes formas de atenção ou sua articulação.

\section{O ponto de vista do usuário indígena}

$\mathrm{Na}$ tentativa de apreender a organização local dos serviços segundo a perspectiva dos doentes ${ }^{3}$, buscou-se identificar os modos como estes acessam as diferentes formas de atenção disponíveis. Para ilustrar tais práticas, será apresentada uma síntese dos dados da observação participante, na forma de itinerário terapêutico ${ }^{8,9}$, que, percorrido pelo sr. Xavier, é o ponto de partida dessa discussão:

O sr. Xavier, morador da aldeia Buriti, teve um episódio de febre persistente. Alguns dias após o início da febre, procurou o AIS de Buriti, que fez uma avaliação inicial e pediu a remoção do paciente para São Gabriel da Cachoeira. O sr. Xavier ficou, então, internado na Casai, sendo atendido no hospital geral local, onde recebeu diagnóstico de dengue e prescrição de paracetamol.

Ao retornar para a aldeia, inicialmente ele teve uma melhora, mas, após ingerir carne de porco, houve uma piora dos sintomas que foi entendida como "a doença não gostou". O sr. Xavier optou, então, por buscar um conhecedor local de plantas medicinais, mas não houve melhora. Pouco tempo depois, o AIS retornou à casa do sr. Xavier e lhe forneceu mais antitérmico. Entretanto, a febre e o mal-estar continuaram, o que levou a mulher do doente a buscar atendimento com outro conhecedor de plantas. Esse segundo conhecedor de plantas faz um diagnóstico de "estrago" e, para curá-lo, buscou no mato uma planta de uso medicinal.

O sr. Xavier disse que no primeiro momento em que aplicou o remédio doeu muito, que ele "ficou louco", mas logo depois melhorou. No segundo dia de tratamento tradicional, o AIS fez nova visita domiciliar e, durante a conversa, concordou com o diagnóstico de "estrago". "O sr. Xavier já se sentia melhor e confiante, apesar da persistência da febre. A partir desse dia, retomou suas atividades cotidianas e considerou sua enfermidade resolvida". (Trecho editado do Diário de Campo)

A partir desse caso, observa-se que o mesmo problema gerou dois diagnósticos: o de dengue e o de estrago, produzidos segundo as diferentes classificações dos sinais e sintomas coexistentes naquele cenário social. No decorrer do itinerário terapêutico, fica claro o confronto e a negociação de diferentes perspectivas sobre saúde e doença, o que demonstra a pertinência e a importância de se discutir a diretriz da atenção diferenciada e suas formas de operacionalização.

Para esse povo indígena, a doença remete a relatos míticos que são constantemente atualizados para explicar o mundo atual. A partir de seus saberes e experiências acumulados, os sujeitos refletem, decidem e manejam, de forma relativamente autônoma, a busca e o uso das diferentes formas de atenção (tradicional e biomédico), enfatizando a importância da autoatenção na construção do itinerário terapêutico.

Nesse contexto, a categoria "estrago" - uma doença tradicional causada por malefício intencional de terceiros ${ }^{12}$ - é percebida tanto como um fruto de ação humana (alguém colocou "veneno") quanto como uma entidade detentora de vontade e capacidade de discriminação dos eventos (a doença "não gostou" da carne de porco comida pelo doente). É um cenário coerente com os achados de Langdon ${ }^{9}$ que destaca o significado da doença como uma construção cultural que remete ao contexto cosmológico, social e moral, que é, por sua vez, atributo causal implicado no surgimento do evento patológico.

No caso relatado, após a consulta com o especialista indígena que identificou a causa da doença, o sr. Xavier considerou-se curado, mesmo com a persistência da febre, tornando evidente que, do ponto de vista Baniwa, o significado da doença extrapola o sintoma físico. O sintoma físico pode ser aliviado pelo uso de medicamen- 
tos industrializados, mas suas causas têm que ser combatidas pelos cuidados tradicionais. A perspectiva do usuário torna possível perceber, na vida cotidiana das pessoas, a articulação de concepções e práticas terapêuticas (como as tradicionais e a biomédica) bastante diversas, reafirmando o pluralismo médico no subsistema e a importância da autoatenção.

É importante também assinalar que, nesse itinerário terapêutico, o AIS propiciou o acesso ao medicamento industrializado e ao tratamento ocidental. Em revisão da literatura Diehl et al. ${ }^{38}$ assinalam que nas aldeias a expectativa sobre o trabalho do AIS é de que dominem a prática biomédica e não as práticas tradicionais de cura. No caso apresentado, entretanto, tanto o doente quanto o AIS transitam entre a medicina ocidental e a indígena, apesar das diferenças epistemológicas entre ambas.

No caso Baniwa, o AIS reconhece e concorda com o diagnóstico de "estrago", algo que é considerado irrelevante para o seu trabalho no DSEIRN, não sendo um evento merecedor nem mesmo de registro no formulário de produção do agente e nem no sistema de informação do subsistema de saúde indígena. Mas, ao facilitar o acesso do doente aos cuidados biomédicos, o AIS tanto agiu em consonância com as expectativas da aldeia quanto efetivou a pluralidade dos recursos de saúde disponíveis no cenário local. Sendo assim, considera-se que a análise do modelo de atenção a partir do ponto de vista dos sujeitos que buscam cuidado ${ }^{3}$, pode-se identificar algumas características essenciais para pensar a atenção diferenciada: o pluralismo médico e a articulação/coexistência entre diferentes formas de atenção.

\section{Considerações Finais}

Com base na análise da Pnaspi distinguiram-se três princípios que direcionam a atenção diferenciada na organização do modelo de atenção do subsistema de saúde indígena: adequação de tecnologias; qualificação dos profissionais para o contexto intercultural; participação indígena. Incorporou-se a perspectiva antropológica de Menéndez para problematizar a formulação e a implementação dessa diretriz.

A formulação do modelo de atenção para os DSEI, ao se restringir às instâncias biomédicas oficiais, ignora e nega a existência de outras formas de atenção nos territórios indígenas. A proposta de "adequar" as tecnologias e qualifi- car os profissionais, de acordo com o contexto local, parte do pressuposto de que se vai "transformar" conhecimentos e práticas hegemônicos, predominantemente biomédicos, nos sistemas de atenção e de formação profissional no Brasil. Menéndez ${ }^{3}$ destaca que a tendência da biomedicina é se apropriar das outras formas de atenção para manter sua hegemonia, e que a partir do ponto de vista dos profissionais de saúde em geral se identifica como contraditória a coexistência de diferentes formas de atenção.

A sobrevalorização da biomedicina e o não reconhecimento de outras formas de atenção são produtos das disputas simbólicas existentes no campo da saúde, sendo que os procedimentos técnico-burocráticos do DSEI reforçam essa legitimação unilateral.

Assim, ao analisar a organização do subsistema a partir do trabalho dos AIS, não foi constatada a inclusão ou o reconhecimento das representações e das práticas indígenas de saúde e doença nas atividades ou nos registros do subsistema. $\mathrm{O}$ pressuposto de que a inclusão de indígenas nas equipes garantiria uma "adequação cultural" das práticas do modelo de atenção se mostrou inválido. A ação do DSEIRN no plano local, desenvolvida pelos AIS, organiza-se a partir de rotinas e tarefas padronizadas para a atenção primária e refletem o padrão biomédico hegemônico na atenção à saúde no SUS. A capacidade dos AIS de interferir e transformar o sistema é muito reduzida, tanto pela hierarquização do trabalho em equipe quanto pela pouca permeabilidade dos procedimentos técnico-burocrático às especificidades culturais.

Apesar desses elementos críticos, é estratégica e fundamental a inserção e ampliação dos profissionais indígenas no sistema, pois a pesquisa evidenciou que os AIS reconhecem as práticas e conhecimentos tradicionais no território.

Já o acompanhamento e a sistematização dos itinerários terapêuticos dos usuários indígenas mostraram, nos territórios indígenas, a existência de uma diversidade de formas de atenção, que incluiu a tradicional, biomédica e autoatenção, e de fluxos no processo de busca por cuidado. Esses processos são conhecidos pelos AIS, de modo que estes podem produzir no cotidiano arranjos para aproximar as práticas do Distrito da autoatenção.

A análise dos modelos a partir do ponto de vista dos sujeitos e grupos sociais, tendo a autoatenção como foco, possibilitou visualizar o pluralismo médico e a articulação entre formas de atenção no DSEIRN. Considera-se que a análise da autoatenção é o espaço privilegiado para 
refletir sobre essas características no subsistema de saúde indígena, de forma que sugere-se outros estudos.

\section{Colaboradores}

ALM Pontes realizou a pesquisa de campo, levantamento documental, análise e interpretação de dados e redação do manuscrito. L Garnelo contribuiu na análise e interpretação de dados e redação do manuscrito. S Rego participou da revisão do manuscrito. Todos os autores revisaram o texto e aprovaram sua versão final.

\section{Agradecimentos}

Artigo produzido no âmbito do Projeto Saúde e Condições de Vida de Povos Indígenas na Amazônia, Programa de Apoio a Núcleos de Excelência - Pronex/Fapeam/CNPq. Também contou com apoio de bolsa Capes (PDSE) no Centro de Investigaciones y Estudios Superiores en Antropología Social (Ciesas/Mx). 


\section{Referências}

1. Brasil. Fundação Nacional de Saúde. Política Nacional de Saúde. Política Nacional de Atenção à Saúde dos Povos Indígenas. Brasília: Ministério da Saúde, Fundação Nacional de Saúde; 2002.

2. Paim JS. A reforma sanitária e os modelos assistenciais. In: Rouquayrol MZ, organizadora. Epidemiologia $e$ Saúde. Rio de Janeiro: Editora Medsi; 1999. p. 473-482.

3. Menéndez E. Modelos de atención de los padecimientos: de exclusiones teóricas y articulaciones prácticas. Cien Saude Colet 2003; 8(1):185-207.

4. Menéndez E. Intencionalidad, experiencia y función: la articulación de los saberes médicos. Desacatos 2005; 14:33-69.

5. Menéndez E. Modelos, saberes y formas de atención de los padecimientos: de exclusiones ideológicas y de articulaciones prácticas. In: Menéndez E. De sujetos, saberes y estructuras: introducción al enfoque relacional en el estudio de la salud colectiva. Buenos Aires: Lugar Editorial; 2009. p. 25-72.

6. Bourdieu P. Coisas ditas. São Paulo: Brasiliense; 2004.

7. Bourdieu P. O poder simbólico. Rio de Janeiro: Bertrand Brasil; 2010.

8. Gerhardt TE. Itinerários terapêuticos em situações de pobreza: diversidade e pluralidade. Cad Saude Publica 2006; 22(11):2449-2463.

9. Langdon EJ. A construção sociocultural da doença e seu desafio para a prática médica. In: Baruzzi RG, Junqueira C, organizadores. Parque Indígena do Xingu: saúde, cultura e histórica. São Paulo: Terra Virgem, Unifesp; 2005.

10. Bonnewitz P. Primeiras lições sobre a sociologia de P. Bourdieu. Petrópolis: Vozes; 2003.

11. Garnelo L. Mito, História e representação social de Doença Sexualmente Transmissível entre os Baniwa - Noroeste Amazônico. In: Nascimento DR, Carvalho DM, Marques RC, organizadores. Uma História Brasileira das Doenças. Rio de Janeiro: Ed. Mauad; 2006.

12. Garnelo L. Poder, Hierarquia e Reciprocidade: saúde e harmonia entre os Baniwa do Alto Rio Negro. Rio de Janeiro: Editora Fiocruz; 2003.

13. Flick U. Desenho da Pesquisa Qualitativa. Porto Alegre: Artmed; 2009.

14. Richardson RJ. Pesquisa social: métodos e técnicas. $3^{\mathrm{a}}$ ed. São Paulo: Atlas; 2012.

15. Chizzotti A. Pesquisa qualitativa em ciências humanas e sociais. Chizzotti A. 3a ed. Petrópolis: Vozes; 2010.

16. Cicourel A. Teoria e método em pesquisa de campo. In: Zaluar A, organizadora. Desvendando máscaras sociais. $2^{\mathrm{a}}$ ed. Rio de Janeiro: Livraria Francisco Alves Editora S.A.; 1980. p. 87-121.

17. Foote-Whyte W. Treinando a observação participante. In: Guimarães AZ, organizadora. Desvendando máscaras sociais. $2^{\text {a }}$ ed. Rio de Janeiro: Livraria Francisco Alves Editora; 1980. p. 77-86.

18. Minayo MCS. O desafio do conhecimento: pesquisa qualitativa em saúde. São Paulo: Hucitec; 2010.
19. Duarte R. Pesquisa Qualitativa: reflexões sobre o trabalho de campo. Cadernos de Pesquisa 2002; 115:139-154.

20. Michelat G. Sobre a utilização da entrevista não-diretiva em Sociologia. In: Thiollent MJM, organizador. Crítica Metodológica, Investigação Social e Enquete Operária. Luanda: Editora Polis; 1982. p. 191-211.

21. Pinto MJ. Comunicação e Discurso: introdução à análise de discursos. São Paulo: Hacker Editores; 2002.

22. Fairclough N. Discurso e mudança social. Brasília: Editora Universidade de Brasília; 2001, 2008 (reimpressão).

23. Araújo IS. A reconversão do Olhar: prática discursiva e produção dos sentidos na intervenção social. São Leopoldo: Unisinos; 2000.

24. Brasil. Fundação Nacional de Saúde. Política Nacional de Saúde. Departamento de Saúde Indígena. A formação de Agentes Indígenas de Saúde no âmbito da Política Nacional de Atenção à Saúde dos Povos Indígenas. Brasil 1999-2005. Brasília: Ministério da Saúde, Fundação Nacional de Saúde; 2005.

25. Hannerz U. Fluxos, Fronteiras, Híbridos: palavras-chave da antropologia transnacional. Mana 1997; 3(1):7-39.

26. Garnelo L, Wright R. Doença, cura e serviços de saúde: representações, práticas e demandas Baniwa. Cad Saude Publica 2001; 17(2):273-284.

27. Novo MP. Saúde e Interculturalidade: a participação dos Agentes Indígenas de Saúde/ AISs do Alto Xingu. Revista de Antropologia Social dos alunos do PPGAS-UFSCar 2009; 1(1):122-147.

28. Langdon EJ, Diehl EE. Participação e autonomia nos espaços interculturais de saúde indígena: reflexões a partir do sul do Brasil. Saúde soc. 2007; 16(2):19-36.

29. Dias-Scopel RP. O agente indígena de saúde Xokleng: por um mediador entre a comunidade indígena e o serviço de atenção diferenciada à saúde - uma abordagem da antropologia da saúde [dissertação]. Florianópolis: Universidade Federal de Santa Catarina; 2005.

30. Rocha ESC. Uma etnografia das práticas sanitárias no distrito sanitário especial indígena do Rio Negro noroeste do Amazonas [dissertação]. Manaus: Universidade Federal do Amazonas; 2007.

31. Kelly JA. State Healthcare and yanomami transformation: a symmetrical anthropology. Tucson: The University of Arizona Press; 2011.

32. Novo MP. Os agentes indígenas de saúde do Alto Xingu. Brasília: Paralelo; 2010.

33. Langdon JE, Diehl EE, Wiik FB, Dias-Scopel RP. A participação dos agentes indígenas de saúde nos serviços de atenção à saúde: a experiência em Santa Catarina, Brasil. Cad Saude Publica 2006; 22(12):2637-2646.

34. Silva Junior AG. Modelos tecnoassistenciais em saúde: $o$ debate no campo da Saúde Coletiva. São Paulo: Hucitec; 2006. 
35. Ayres JRCM. Organização das Ações de Atenção à Saúde: modelos e práticas. Saúde soc 2009; 18(Supl. 2):11-23.

36. Erthal RMC. A formação do agente de saúde indígena Tikúna no Alto Solimões: uma avaliação crítica. In: Coimbra Júnior C, Santos RV, Escobar AL. Epidemiologia e saúde dos povos indígenas no Brasil. Rio de Janeiro: Editora Fiocruz; 2003. p. 197-216.

37. Mendonça SBM. O agente indígena de saúde no Parque Indígena do Xingu: Reflexões. In: Baruzzi RG, Junqueira C, organizadores. Parque Indígena do Xingu: Saúde, Cultura e História. São Paulo: Terra Virgem, Unifesp 2005. p. 227-246.

38. Diehl EE, Langdon EJ, Dias-Scopel RP. Contribuição dos agentes indígenas de saúde na atenção diferenciada à saúde dos povos indígenas brasileiros. Cad Saude Publica 2012; 28(5):819-831.

Artigo apresentado em 12/03/2014

Aprovado em 29/01/2015

Versão final apresentada em 31/01/2015 